\title{
Utility of the waist-to-height ratio, waist circumference and body mass index in the screening of metabolic syndrome in adult patients with type 1 diabetes mellitus
}

\author{
Aldo Ferreira-Hermosillo ${ }^{1}$, Claudia Ramírez-Rentería', Victoria Mendoza-Zubieta ${ }^{2}$ and Mario A Molina-Ayala ${ }^{2 *}$
}

\begin{abstract}
Background: The incidence of macrovascular complications and morbidities associated to metabolic syndrome are increasing in patients with type 1 diabetes mellitus (T1DM). The combination of T1DM with features of insulin resistance similar to that of type 2 diabetes (T2DM), sometimes called "double diabetes", has been associated with central obesity. Since the most methods to accurately detect body fat and insulin resistance are not readily available, we propose that certain indirect indexes for detecting obesity as waist-to-height ratio, waist circumference and body mass index, may be useful when screening for metabolic syndrome in patients with T1DM.

Methods: We performed a transversal evaluation (clinical and biochemical) in all the patients of the T1DM Clinic $(n=120)$. We determined the presence of metabolic syndrome according to the Joint Statement Criteria by the American Heart Association/ National Heart Lung and Blood Institute and the International Diabetes Federation and the utility of certain anthropometric indexes for predicting double diabetes was evaluated.

Results: Thirty seven percent of the patients were considered to have metabolic syndrome using these criteria $(n=30)$. These patients were significantly older $(p=0.002)$, have a higher glycated hemoglobin $(p=0.036)$, cholesterol $(p<0.012)$ and triglyceride concentration $(p<0.01)$ as well as body mass index $(p=0.004)$, waist circumference $(p=0.01)$ and waist-to-height ratio $(p<0.01)$ than the group without metabolic syndrome. Also their $c-H D L$ is lower $(p<0.01)$. A value of 0.52 for waist-to-height ratio correctly classified the largest number of patients (68\% of correctly classified) well as the waist circumference ( $66 \%$ of correctly classified) with an adequate specificity and sensibility. Meanwhile the most precise body mass index value only classified correctly to $61 \%$ of patients.
\end{abstract}

Conclusion: Our data show that waist circumference and waist-to-height ratio indexes are useful to predict the presence of metabolic syndrome in adult patients with type 1 diabetes mellitus.

Keywords: Metabolic syndrome, Type 1 diabetes, Waist circumference, Waist-to-height ratio

\section{Background}

Type 1 diabetes mellitus (T1D) is an autoimmune disease in which pancreatic $\beta$ cells are destroyed, generating an incapacity to maintain appropriate insulin and glucose concentrations [1]. The Instituto Mexicano del Seguro Social (IMSS) is a governmental institution that

\footnotetext{
* Correspondence: mmol_17@yahoo.com.mx

${ }^{2}$ Endocrinology Department, Hospital de Especialidades Centro Médico Nacional Siglo XXI, Instituto Mexicano del Seguro Social (IMSS), Cuauhtémoc No. 330, Colonia Doctores, México City, DF, Mexico

Full list of author information is available at the end of the article
}

provides medical health for approximately 30\% of the Mexican population. One of its recent publications reported a significant increase in the incidence of type 1 diabetes in the population evaluated by this healthcare system. Between the years 2000 and 2010, for the group under 19 years of age, the incidence increased from 3.4 to 6.2 per 100,00 in both sexes $(\mathrm{p}<0.001)$ [2].

Diabetes is one of the main causes of incapacity worldwide and affects all age groups. Years ago, microvascular complications, such as nephropathy, constituted the main cause of morbidity and mortality among patients 
with T1D [3]. More recently, there has been an increase in the macrovascular complication rate, associated with the presence of other cardiovascular risk factors such as hypertension, hypertriglyceridemia, hypoalphalipoproteinemia and central obesity, all of them in the context of metabolic syndrome (MS) [4]. Mexico has one of the largest prevalence in obesity worldwide, which has also affected the population with T1D [5]. Teupe and Bergis described the presence of pathologies commonly associated with type 2 diabetes in patients with T1D and named them "double diabetes" [6]. Even when there have been controversies regarding the use of this term and its diagnosis, these associations are being reported frequently. The treatment costs and complications will theoretically increase in the next years in patients fulfilling the criteria for MS. Accurate screening, early diagnosis and prevention will be important interventions for this group in the upcoming years.

The pathophysiology of MS is complex and it is yet being investigated. It has been hypothesized that the increased visceral adiposity in central obesity is a cardinal factor. Fat is a highly metabolic tissue that generates adipokines, greatly responsible for the development of insulin resistance [7]. Specific methods for visceral adiposity quantification such as axial tomography, magnetic resonance and electric bioimpedance are expensive and, for the most part, inaccessible in developing countries [8]. Therefore, surrogate measurements such as body mass index (BMI) and waist circumference (WC) have been used to assess the risk of MS [9]. All of them have serious limitations: race and clinical characteristics are two of the major determinants for the different results published by each author, making it necessary to validate every index for our specific population.

The waist-to-height ratio (WHtR) has proved to be a better discriminator for the metabolic and cardiovascular risk factors, however, studies differ in their cut-off values and usefulness to detect MS in general population [10]. To our knowledge, this information is not yet available for the population with T1D.

The purpose of this study was to evaluate and compare the utility of WHtR, BMI and WC in the detection of MS in patients with T1D and proposing specific cutoff values for the Mexican population.

\section{Methods}

We performed a transversal evaluation in all the patients in the Type 1 Diabetes Clinic (Hospital de Especialidades Centro Médico Nacional Siglo XXI, tertiary referral center). We included 120 patients with the following characteristics: 18 years of age or older, at least 3 regular yearly visits to the clinic, no infections recorded in the 3 months previous to the study and no change in insulin dose during the same time. Patients with incomplete records or follow-ups, poor treatment adherence and primary dyslipidemias were excluded. The study completed all the requirements of the local ethics committee (Comite Local de Investigación y Ética en Investigación en Salud). The protocol and the aim of the study were fully explained to the subjects, who gave their written consent.

\section{Diagnostic criteria for MS}

We used the joint statement criteria by the American Heart Association/National Heart Lung and Blood Institute (AHA/NHLBI) and the International Diabetes Federation (IDF) [11]. Patients were considered to have MS with 3 or more of the following components: serum triglycerides $>150 \mathrm{mg} / \mathrm{dl}(1.7 \mathrm{mmol} / \mathrm{l})$, serum high-density lipoproteins cholesterol (c-HDL) $<40 \mathrm{mg} / \mathrm{dl}$ $(1.03 \mathrm{mmol} / \mathrm{l})$ in men and $<50 \mathrm{mg} / \mathrm{dL}(1.29 \mathrm{mmol} / \mathrm{l})$ in women or a previously treated dyslipidemia, arterial blood pressure $>130 / 85 \mathrm{mmHg}$ in two different determination or patients receiving treatment with antihypertensive drugs. WC $>90 \mathrm{~cm}$ in men and $>80$ in women. Since patients were treated for type 1 diabetes, they all fulfilled the criteria of fasting plasma glucose $>100 \mathrm{mg} / \mathrm{dl}(5.6 \mathrm{mmol} / \mathrm{l})$ at least once.

\section{Anthropometric measurements}

At the time of evaluation we registered weight (kilograms) and height (meters), as well as WC (centimeters). A single investigator, using the same calibrated scale with an integrated stadiometer, performed anthropometric measurements. WC was determined at the middle point between the inferior rim of the last costal arch and the superior rim of the anterosuperior iliac spine. BMI was calculated as the weight divided by height to the square. Blood pressure was determined in the left arm, after 10 minutes in a resting position, during a fasting state, without coffee or tobacco ingestion in the last week. The sphygmomanometer was calibrated and values were averaged after 2 different measurements with a 5-minute difference between them.

\section{Biochemical determinations}

Laboratory results were obtained with a $6 \mathrm{~mL}$ sample in BD Vacutainer (BD Franklin Lakes, New Jersey USA), centrifuged at $3150 \times g$ for 15 minutes and serum was then analyzed with a kit for glucose, cholesterol, c-HDL and triglycerides (COBAS 2010 Roche Diagnostics, Indianapolis USA) using photocolorimetry with spectrophotometer Roche Modular P800 (2010 Roche Diagnostics, Indianapolis USA). c-HDL samples were treated with enzymes modified with polyethileneglycol and dextrane sulphate, analyzed with the same photocolorimetric technique. Glycated hemoglobin (HbA1c) was evaluated by turbidimetric immunoanalysis (COBAS 2010 Roche Diagnostics, Indianapolis USA). c-LDL was 
calculated with Friedewald formula: c-LDL $(\mathrm{mg} / \mathrm{dl})=$ CT $\mathrm{mg} / \mathrm{dl}$ - (c-HDL $\mathrm{mg} / \mathrm{dl}+$ triglycerides $\mathrm{mg} / \mathrm{dl} / 5)$ if triglycerides were $<400 \mathrm{mg} / \mathrm{dl}[12]$.

\section{Statistic analysis}

Data were analyzed with STATA v.11. KolmogorovSmirnov test was used to determine normality. Results are expressed accordingly with means and standard deviations (SD) or medians and interquartile ranges (IQR). To establish associations between quantitative variables, Student $\mathrm{t}$ test or Mann-Whitney $\mathrm{U}$ test were used. Qualitative variables were associated with $\mathrm{X}^{2}$ or Fisher tests. Receiver Operating Characteristic curves (ROC) for the different indexes and their combinations were used to identify the best cut of point with $95 \%$ confidence intervals. The areas under the curve (AUC) were compared with $\mathrm{X}^{2}$. Additionally correlations where performed using Pearson test. A $p<0.05$ was considered to be significant.

\section{Results}

\section{Baseline characteristics}

Thirty seven percent of the patients were considered to have MS using these criteria $(n=30)$. Table 1 compares the baseline characteristics of the populations with and without MS. Patients with MS are significantly older, have higher BMI, WC and WHtR than the group without MS. Also, their HbA1c, cholesterol and triglyceride concentrations are higher while c-HDL is lower. This group had higher prevalence of hypertension and dyslipidemia.

We performed a ROC curve for WHtR, BMI and WC in our population to determine the best cut off points to predict MS in our population (Figure 1).

Table 2 depicts the best cut off values, sensibility, specificity, likelihood ratio positive $(\mathrm{LR}+)$ and negative (LR-) predictive values and the percentage of correctly classified patients with each index. Likelihood ratios were used for assessing the value of performing these tests. A LR + higher than 1 indicates the test could be used to confirm the disease while a lower LR-indicates the test could be used to rule out the disease. The cutoff points were selected using Youden index, at which (sensitivity + specificity -1 ) is maximized [13]. A value of 0.52 for WHtR correctly classifies the largest number of patients with an adequate specificity and sensibility. This cut off was also appropriate for both sexes. When stratified, the male sub group had an AUC of 0.67 (0.48 - 0.85), sensibility of $62 \%$, specificity of $61 \%$ and also $61 \%$ of correctly classified patients. In female patients the AUC was 0.79 (0.69 - 0.88), sensibility was $74 \%$, specificity $68 \%$ and $70 \%$ of patients classified correctly (complementary data not shown). According to our results the WC cutoff value of $85 \mathrm{~cm}$ has lower sensitivity, specificity and detects fewer
Table 1 Baseline characteristics of the population comparing patients with metabolic syndrome (MS) and without MS

\begin{tabular}{|c|c|c|c|}
\hline Parameter & $\begin{array}{l}\text { With MS } \\
(\mathrm{n}=30)\end{array}$ & $\begin{array}{l}\text { Without MS } \\
\quad(n=90)\end{array}$ & p \\
\hline Age (years) & $35(26-42)$ & $28(20-34)$ & $0.002^{*}$ \\
\hline Evolution of T1D (years) & $20(14.7-28)$ & $16(11-25)$ & 0.051 \\
\hline \multicolumn{4}{|l|}{ Gender } \\
\hline Female & $26 \%$ & $74 \%$ & \multirow{2}{*}{0.584} \\
\hline Male & $12 \%$ & $88 \%$ & \\
\hline Smoking status & $5 \%$ & $18 \%$ & 0.551 \\
\hline Insulin dose (U/day) & $49(40-69.8)$ & $45.5(39-57)$ & $0.086^{*}$ \\
\hline (U/kg/day) & $0.69(0.52-0.98)$ & $0.75(0.59-0.97)$ & 0.974 \\
\hline Weight (m) & $1.62(1.57-1.70)$ & $1.66(1.55-1.67)$ & 0.269 \\
\hline BMI $\left(\mathrm{kg} / \mathrm{m}^{2}\right)$ & $25(23.8-28.7)$ & $23.3(21.3-26.4)$ & $0.004^{*}$ \\
\hline WC (cm) & $90.4 \pm 10.7$ & $79.6 \pm 8.2$ & $<0.01^{\dagger}$ \\
\hline Male & $92.1 \pm 12.6$ & $85.2 \pm 6.1$ & 0.032 \\
\hline Female & $89.6 \pm 10.3$ & $77.2 \pm 7.88$ & $<0.01$ \\
\hline WHtR & $0.54(0.51-0.60)$ & $0.49(0.46-0.53)$ & $<0.001^{*}$ \\
\hline Hypertension & $14 \%$ & $8 \%$ & $<0.01^{\ddagger}$ \\
\hline Dyslipidemia & $18 \%$ & $16 \%$ & $0.02^{\ddagger}$ \\
\hline Glycated hemoglobin (\%) & $8.8(8-10)$ & $8.2(7.5-9.3)$ & $0.036^{*}$ \\
\hline Total colesterol (mg/dl) & $188(169-210)$ & $174.5(153-206)$ & $<0.012^{*}$ \\
\hline Tryglycerides (mg/dl) & $175(136-248)$ & $88(66-113)$ & $<0.01^{*}$ \\
\hline $\mathrm{c}-\mathrm{HDL}(\mathrm{mg} / \mathrm{dl})$ & $46(37-53)$ & $57(48-66)$ & $<0.01^{*}$ \\
\hline Male & $41(37-48)$ & $49(44-60)$ & 0.044 \\
\hline Female & $47(37-55)$ & $60(53-69)$ & $<0.01$ \\
\hline
\end{tabular}

Results are reported as Mean \pm SD or mean and IQR.

*Mann-Whitney $U$ test.

${ }^{\dagger}$ Student $t$ test.

${ }^{\mp} \mathrm{X}^{2}$ test.

patients with MS compared to WHtR. Nevertheless, using cutoff values of $90 \mathrm{~cm}$ for men and $80 \mathrm{~cm}$ for women as recommended by IDF, increases the number of patients correctly classified to 76 and $73 \%$, respectively.

Considering that the patients with MS are older than patients without MS, we performed a Pearson correlation test. This test showed a small but positive correlation between age and WC $(\rho=0.217 ; p=0.017)$ but there was no correlation with WHtR index $(p=0.063)$. Nevertheless, there was a positive correlation in patients under 30 years of age $(p=0.261, p=0.039)$ which is not observed in older patients $(\mathrm{p}=0.773)$. We didn't observe any direct association between gender and MS even after dividing by age groups. Nevertheless if WC cutoff points are adjusted to gender, it reaches a MS detection of $76 \%$ for male and $73 \%$ for female.

Regarding the predictive power of BMI to detect MS, we found that the traditional cutoff value used to predict excess weight $\left(\mathrm{BMI}>25 \mathrm{~kg} / \mathrm{m}^{2}\right)$ had a sensibility of only $57 \%$ 


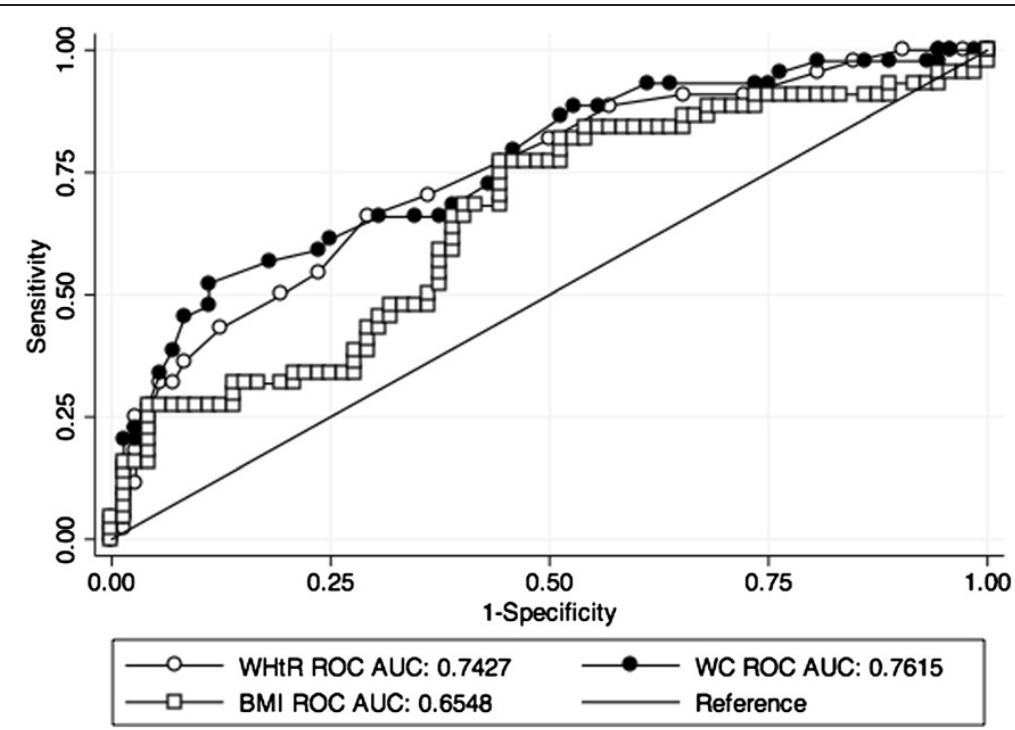

Figure 1 ROC curves for WC, WHtR and BMI. The ROC curves were performed to evaluate the best cut-off value for predicting metabolic syndrome in our population. $\mathrm{WC}=$ waist circumference, $\mathrm{WHtR}=$ waist-to-height ratio and $\mathrm{BMI}=$ body mass index.

a specificity of $64 \%$ and a precision of $61 \%$, while the cut off for obesity $\left(\mathrm{BMI}>30 \mathrm{~kg} / \mathrm{m}^{2}\right)$ had a lower sensibility (13\%) but a high specificity (98\%) with a precision of $67 \%$.

These indexes may be proposed as one more of the screening tools easily available for clinicians in all centers. However, no single index seems to provide by itself the combination of high sensibility and specificity required for most diagnostic tests. However, the combination of these indexes may be helpful. For instance, combining the WHtR cutoff point of 0.52 with the BMI cutoff point of $24 \mathrm{~kg} / \mathrm{m}^{2}$ presented a sensibility of $59 \%$, but increased specificity to $83 \%, \mathrm{LR}(+) 3.45, \mathrm{LR}(-) 0.5$ and a precision of $77 \%$. When we analyzed the commonly used WHtR cutoff point of 0.5 and the cutoff of value for BMI of $25 \mathrm{~kg} / \mathrm{m}^{2}$ we observed a lower sensibility of $52 \%$ with a similar high specificity of $81 \%, \operatorname{LR}(+)$ 2.72, $\operatorname{LR}(-) 0.59$ and a precision of $74 \%$.

On the contrary, combining the WHtR cutoff point of 0.5 with the BMI cutoff point for obesity $\left(30 \mathrm{~kg} / \mathrm{m}^{2}\right)$, increases sensibility to $86 \%$ with a lower, but useful specificity of $66 \%, \mathrm{LR}(+) 2.55, \mathrm{LR}(-) 0.22$, however precision is only $14 \%$.
Finally, using the $\chi^{2}$ test to evaluate the differences between the AUC, we found that when we used BMI as a reference parameter, both WHtR and WC predicted MS better (AUC BMI 0.65 vs AUC WHtR 0.74, $\mathrm{p}=0.028$ and AUC WC 0.76, $\mathrm{p}=0.017$ ). We didn't find any statistically significant difference between WHtR and WC $(\mathrm{p}=0.44)$.

\section{Discussion}

Our study included a total of 120 adult patients with T1D (>18 years old) where we found a shocking $37 \%$ of patients fulfilling current criteria for MS using current diagnostic tools. Eighteen percent had dyslipidemia and $14 \%$ were hypertensive.

Recent data show that diagnosing and treating MS should be new concern for clinicians treating T1DM populations, considering the possibility of higher cardiovascular risk associated with it; however, the stratification is currently based on laboratory data, after the biochemical alterations are present [14]. To date, there is no single clinical, inexpensive, easily available index to predict the presence of MS in T1DM patients. Clinical

Table 2 Best cutoff values to predict metabolic syndrome

\begin{tabular}{lccccccc}
\hline Index & AUC (IC 95\%) & Cut off value & Sensibility & Specificity & \% of correctly classified patients & LR+ & LR- \\
\hline WHtR & $0.75(0.66-0.84)$ & 0.52 & $70 \%$ & $66 \%$ & $68 \%$ & 2.05 & 0.44 \\
WC & $0.76(0.67-0.85)$ & $85 \mathrm{~cm}$ & $66 \%$ & $65 \%$ & $66 \%$ & 0.52 \\
Men & $0.71(0.49-0.93)$ & $90 \mathrm{~cm}$ & $69 \%$ & $81 \%$ & $76 \%$ & 0.89 \\
Women & $0.80(0.70-0.90)$ & $80 \mathrm{~cm}$ & $90 \%$ & $63 \%$ & $73 \%$ & 3.63 \\
BMl & $0.66(0.56-0.76)$ & $24 \mathrm{~kg} / \mathrm{m}^{2}$ & $78 \%$ & $58 \%$ & $61 \%$ & 0.38 \\
\hline
\end{tabular}

LR+: positive likelihood ratio. LR-: negative likelihood ratio. WHtR: waist-to-height ratio. WC: waist circumference. BMI: body mass index. 
data may help clinicians distinguish high-risk patients that may be candidates for additional interventions and studies. Primary prevention and low costs are important concerns for these decisions, especially in developing countries. Simple, but validated anthropometric measurements may be useful tools for these ends [15].

Our results are different from our previously reported data where the prevalence was $25 \%$ using the National Cholesterol Education Program: Adult Treatment Panel III (NCEP:ATPIII) criteria [16] and with the results of Thorn, et al. in the Finnish population (38\% men, 40\% women) [17]. We should consider the difficulties and controversies defining MS in T1DM patients and the limitations of using only clinical and general laboratory data in our study. Since all the patients had elevated serum glucose at least once, and this parameter is included as a diagnostic criterion, it could overestimate the prevalence of MS but if it is not considered, MS could be underestimated. However the same limitation will affect other publications if no other accurate system is proposed for the specific T1DM population.

Determining insulin resistance may also prove difficult in these patients. The gold standard to assess insulin resistance is the hyperinsulinemic euglycemic clamp. [18] However, this procedure is complex, expensive and it is usually not performed as part of the everyday evaluation of patients. It has been proposed that insulin dose could discriminate patients with insulin resistance; however, this is unreliable because it is inaccurately reported by patients and the potential effect of residual endogenous insulin secretion [19]. Nowadays, eGDR (estimated glucose disposal rate) index has been proposed for determining insulin resistance in patients with T1D. This study appears to adequately correlate with the clamp technique. [20] At this time, studies are being carried out to calculate specific cutoff points for this index in the Mexican population.

Clearly, MS in T1DM was a fairly unknown phenomenon until recent years. Along with the increasing incidence in obesity, T1DM patients are developing more features previously associated only with T2DM and may have higher cardiovascular risk in the future. A study in the Pittsburgh Epidemiology of Diabetes Complications (EDC) cohort studied 589 patients and showed a change in the BMI of T1DM patients in the last 20 years [21]. They found that obesity (BMI $>30 \mathrm{~kg} / \mathrm{m}^{2}$ ) rose up to $22.7 \%$ and overweight population (BMI $25-30 \mathrm{~kg} / \mathrm{m}^{2}$ ) increased from 26 to a staggering $42 \%$ [22]. In the Mexican population, this increased weight has been attributed to two different but related factors: an exposure to calorie-rich diets and the over-insulinization associated with the intensive control therapies for T1D [23].

We don't know yet the exact mechanisms by which MS increases the risk of cardiovascular events. Obesity may be a central factor in the pathophysiology of "double diabetes". Some theories propose the participation of inflammatory cytokines secreted by the adipose tissue, known to generate atherothrombosis in type 2 diabetes [24-26]. Other studies have associated the increasing insulin concentrations to the stimulation of $11 \beta$ - hydroxysteroid dehydrogenase, which modifies the differentiation from fibroblasts to adipocytes and transformation from cortisone to the highly active cortisol [27].

Clinical data is important to detect risk factors for cardiovascular disease. BMI is one of the most frequently used indexes to detect obesity, however it can't discriminate between excess adiposity and muscle mass [28] and it does not detect abdominal obesity. In our study we found that the traditionally used cut-off values to detect obesity had low sensibilities for MS. Another index, the WC correlates better with the abdominal adipose tissue and has been successfully associated with cardiovascular risk by different authors [29]. This index, however, may underestimate the real cardiovascular risk in patients with small stature, which may be important in many groups, such as ours [30].

It is still controversial which of the tree indexes is better predicting the development of MS. A Chinese study found that these three markers were useful to detect MS [31]. In the San Antonio Heart Study, BMI and WC were found to be equally useful in the Mexican-american population [32]. The INTERHEART study [33] and Bener, et al. [34] found that WC was the best index, while Ashweel, et al. found in 31 studies that WHtR was the best predictor [35]. It would seem that different ethnic groups require different indexes and that cut-off values must be standardized for each one of them.

Regarding the Mexican population, Elizondo-Montemayor, et al. found that children aged 6-12 years with the diagnosis of overweight and obesity had a $23 \%$ of MS with a cut off value of $0.59 \mathrm{LR}(+) 3.80, \mathrm{LR}(-)$ 0.232, sensibility of $81.8 \%$ and specificity of $78.5 \%$, in contrast with the value of 0.50 found in the literature, which is supposed to have a sensibility of $100 \%$, with a specificity of only $22.7 \%, \mathrm{LR}(+) 1.29$ and $\mathrm{LR}(-) 0.000$ [36]. The same study showed that the $90^{\text {th }}$ percentile of WC had a precision of $82.6 \%$, while the $97^{\text {th }}$ percentile of BMI had a precision of $76 \%$.

However, none of these results reflects the adult population with T1D, which tends to be more obese. Our results show that there is a correlation between some of these indexes and age, but only in patients under 30 years of age. This may be due to the fact that all older patients tend to gain weight whether they develop MS or not. This may also mean that it could be more difficult to detect MS in T1DM patients as age increases and it confirms the need for preventive interventions at young ages.

In the current study, we found WC and WHtR to be the best predictive indexes for MS with a cut-off value 
of 0.52 (precision: 68\%). Also, the proposed values for WC were highly sensitive and specific.

The preference of sensitivity $v s$ specificity depends on the objective of the diagnostic tool. Most screening tools are selected when they are highly sensitive because of the reduced chance of false negative results and they are very useful when screening open populations, however higher specificities are required when we want to determine which patients should be referred for further evaluation given the high probabilities of MS. The final choice of one over the other may be in the hands of the clinician when a specific case presents before him.

On the other hand, for patients with a BMI over $24 \mathrm{~kg} / \mathrm{m}^{2}$, the precision is high and using this index as a gold standard WC and WHtR are better markers for MS. This BMI cutoff is lower than the one proposed internationally to diagnose the overweight population, which suggests that our group may require a different set of diagnostic indexes in order to predict their cardiovascular risk.

This study is the first one to describe the utility of these indexes in adult patients with T1D. Using a clinical index is not a substitute for laboratory and specialized workup, however, when properly validated, may be a useful tool for clinicians worldwide. We propose that the combination of these simple indexes may be helpful for this and other larger populations similar to ours. Prospective studies with larger cohorts may be necessary to determine if it is applicable in other ethnic groups, their specific cut off points and the differentiation between sexes and age groups.

\section{Conclusion}

WC and WHtR indexes are useful to predict the presence of metabolic syndrome in adult patients with T1D. We found that the WHtR index and WC allow for discrimination of patients with T1D and an increased probability of metabolic syndrome. The cut-off value of WHtR of 0.52 was the most accurate (68\%) to discriminate MS in Mexican T1D. In ethnic groups where height variations could interfere with the precision of other indexes, WHtR could be a simple and useful tool to screen for cardiovascular risk factors in type 1 diabetes population.

\footnotetext{
Abbreviations

AHA/NHLBI: American Heart Association/National Heart Lung and Blood Institute; AUC: Area under curve; BMI: Body mass index; C-HDL: Cholesterol associated to high density lipoproteins; C-LDL: Cholesterol associated to low density lipoproteins; HbA1c: Glycated hemoglobin a1c; IDF: International Diabetes Federation; IMSS: Instituto Mexicano del Seguro Social, Mexican Institute of Social Security; IQR: Interquartile ranges; LR: Likelihood ratio; MS: Metabolic syndrome; NCEP:ATPIII: National Cholesterol Education Program: Adult Treatment Panel III; SD: Standard deviations; T1D: Type 1 diabetes mellitus; WC: Waist circumference; WtHR: Waist-to-height ratio.
}

\section{Competing interests}

The authors declare that they have no competing interests.

\section{Authors' contributions}

AFH and MAMA participated in the collection and analysis of data and in the writing of the manuscript. CRR and VMZ participated in the analysis of data and in the writing of the manuscript. All authors have read and approved the final version of the manuscript.

\section{Author details}

${ }^{1}$ Endocrinology Experimental Investigation Unit, Hospital de Especialidades Centro Médico Nacional Siglo XXI, Instituto Mexicano del Seguro Social (IMSS), Cuauhtémoc No. 330, Colonia Doctores, México City, DF, Mexico. ${ }^{2}$ Endocrinology Department, Hospital de Especialidades Centro Médico Nacional Siglo XXI, Instituto Mexicano del Seguro Social (IMSS), Cuauhtémoc No. 330, Colonia Doctores, México City, DF, Mexico.

Received: 25 November 2013 Accepted: 26 February 2014 Published: 4 March 2014

\section{References}

1. Wherrett DK, Daneman D: Prevention of type 1 diabetes. Endocrinol Metab Clin North Am 2009, 38(4):777-790.

2. Gomez-Diaz RA, Perez-Perez G, Hernandez-Cuesta IT, Rodriguez-Garcia Jdel C, Guerrero-Lopez R, Aguilar-Salinas CA, Wacher NH: Incidence of type 1 diabetes in Mexico: data from an institutional register 2000-2010. Diabetes Care 2012, 35(11):e77.

3. Chillaron JJ, Flores-Le-Roux JA, Goday A, Benaiges D, Carrera MJ, Puig J, Cano-Perez JF, Pedro-Botet J: Metabolic syndrome and type-1 diabetes mellitus: prevalence and associated factors. Rev Esp Cardiol 2010, 63(4):423-429.

4. Reaven GM: Banting lecture 1988. Role of insulin resistance in human disease. Diabetes 1988, 37(12):1595-1607.

5. Barrera-Cruz A, Avila-Jimenez L, Cano-Perez E, Molina-Ayala MA, Parrilla-Ortiz Jl, Ramos-Hernandez Rl, Sosa-Caballero A, Sosa-Ruiz Mdel R, Gutierrez-Aguilar J: Practice clinical guideline. Prevention, diagnosis and treatment of overweight and obesity. Rev Med Inst Mex Seguro Soc 2013, 51(3):344-357.

6. Teupe B, Bergis K: Epidemiological evidence for "double diabetes". Lancet 1991, 337(8737):361-362.

7. Waki H, Tontonoz P: Endocrine functions of adipose tissue. Annu Rev Pathol 2007, 2:31-56.

8. Shuster A, Patlas M, Pinthus JH, Mourtzakis M: The clinical importance of visceral adiposity: a critical review of methods for visceral adipose tissue analysis. Br J Radiol 2012, 85(1009):1-10.

9. Schneider HJ, Glaesmer H, Klotsche J, Bohler S, Lehnert H, Zeiher AM, Marz W, Pittrow D, Stalla GK, Wittchen HU: Accuracy of anthropometric indicators of obesity to predict cardiovascular risk. J Clin Endocrinol Metab 2007, 92(2):589-594.

10. Ashwell M, Gibson S: Waist to height ratio is a simple and effective obesity screening tool for cardiovascular risk factors: analysis of data from the British National Diet And Nutrition Survey of adults aged 19-64 years. Obes Facts 2009, 2(2):97-103.

11. Alberti KG, Eckel RH, Grundy SM, Zimmet PZ, Cleeman Jl, Donato KA Fruchart JC, James WP, Loria CM, Smith SC Jr: Harmonizing the metabolic syndrome: a joint interim statement of the International Diabetes Federation Task Force on Epidemiology and Prevention; National Heart, Lung, and Blood Institute; American Heart Association; World Heart Federation; International Atherosclerosis Society; and International Association for the Study of Obesity. Circulation 2009, 120(16):1640-1645.

12. Friedewald WT, Levy RI, Fredrickson DS: Estimation of the concentration of low-density lipoprotein cholesterol in plasma, without use of the preparative ultracentrifuge. Clin Chem 1972, 18(6):499-502.

13. Akobeng AK: Understanding diagnostic tests 3 : receiver operating characteristic curves. Acta Paediatr 2007, 96(5):644-647.

14. Cleland SJ: Cardiovascular risk in double diabetes mellitus-when two worlds collide. Nat Rev Endocrinol 2012, 8(8):476-85.

15. Berber A, Gomez-Santos R, Fanghanel G, Sanchez-Reyes L: Anthropometric indexes in the prediction of type 2 diabetes mellitus, hypertension and dyslipidaemia in a Mexican population. Int J Obes Relat Metab Disord 2001, 25(12):1794-1799.

16. Ferreira Hermosillo A, Vargas Ortega G, Gonzalez Virla B, Mercado Atri M, Molina Ayala M: Prevalence of metabolic syndrome (MS) in patients with type 1 diabetes (DM1). Gac Med Mex 2012, 148(2):137-143. 
17. Thorn LM, Forsblom C, Waden J, Saraheimo M, Tolonen N, Hietala K, Groop PH: Metabolic syndrome as a risk factor for cardiovascular disease, mortality, and progression of diabetic nephropathy in type 1 diabetes. Diabetes Care 2009, 32(5):950-952.

18. Radikova Z: Assessment of insulin sensitivity/resistance in epidemiological studies. Endocr Regul 2003, 37(3):189-194.

19. Cleland SJ, Fisher BM, Colhoun HM, Sattar N, Petrie JR: Insulin resistance in type 1 diabetes: what is 'double diabetes' and what are the risks? Diabetologia 2013, 56(7):1462-1470.

20. Williams KV, Erbey JR, Becker D, Arslanian S, Orchard TJ: Can clinical factors estimate insulin resistance in type 1 diabetes? Diabetes 2000, 49(4):626-632.

21. Pambianco G, Costacou T, Ellis D, Becker DJ, Klein R, Orchard TJ: The 30-year natural history of type 1 diabetes complications: the Pittsburgh Epidemiology of Diabetes Complications Study experience. Diabetes 2006, 55(5):1463-1469.

22. Conway B, Miller RG, Costacou T, Fried L, Kelsey S, Evans RW, Orchard TJ: Temporal patterns in overweight and obesity in type 1 diabetes. Diabet Med 2010, 27(4):398-404.

23. Purnell JQ, Hokanson JE, Marcovina SM, Steffes MW, Cleary PA, Brunzell JD: Effect of excessive weight gain with intensive therapy of type 1 diabetes on lipid levels and blood pressure: results from the DCCT. Diabetes Control and Complications Trial. JAMA 1998, 280(2):140-146.

24. Jager A, van Hinsbergh WW, Kostense PJ, Emeis JJ, Nijpels G, Dekker JM, Heine RJ, Bouter LM, Stehouwer CD: Increased levels of soluble vascular cell adhesion molecule 1 are associated with risk of cardiovascular mortality in type 2 diabetes: the Hoorn study. Diabetes 2000, 49(3):485-491.

25. Jager A, van Hinsbergh WW, Kostense PJ, Emeis JJ, Yudkin JS, Nijpels G, Dekker JM, Heine RJ, Bouter LM, Stehouwer CD: von Willebrand factor, C-reactive protein, and 5-year mortality in diabetic and nondiabetic subjects: the Hoorn Study. Arterioscler Thromb Vasc Biol 1999, 19(12):3071-3078.

26. Schulze MB, Shai I, Rimm EB, Li T, Rifai N, Hu FB: Adiponectin and future coronary heart disease events among men with type 2 diabetes. Diabetes 2005, 54(2):534-539.

27. Rodrigues TC, Canani LH, Gross JL: Metabolic syndrome, insulin resistance and cardiovascular disease in type-1 diabetes mellitus]. Ara Bras Cardiol 2010, 94(1):134-139.

28. Hall DM, Cole TJ: What use is the BMI? Arch Dis Child 2006, 91(4):283-286.

29. Ness-Abramof R, Apovian CM: Waist circumference measurement in clinical practice. Nutr Clin Pract 2008, 23(4):397-404.

30. Hsieh $\mathrm{SD}$, Yoshinaga $\mathrm{H}$ : Do people with similar waist circumference share similar health risks irrespective of height? Tohoku J Exp Med 1999, 188(1):55-60.

31. Liu Y, Tong G, Tong W, Lu L, Qin X: Can body mass index, waist circumference, waist-hip ratio and waist-height ratio predict the presence of multiple metabolic risk factors in Chinese subjects? BMC Public Health 2011, 11:35.

32. Han TS, Williams K, Sattar N, Hunt KJ, Lean ME, Haffner SM: Analysis of obesity and hyperinsulinemia in the development of metabolic syndrome: San Antonio Heart Study. Obes Res 2002, 10(9):923-931.

33. Schneider HJ, Klotsche J, Stalla GK, Wittchen HU: Obesity and risk of myocardial infarction: the INTERHEART study. Lancet 2006 367(9516):1052. author reply 1054.

34. Bener A, Yousafzai MT, Darwish S, Al-Hamaq AO, Nasralla EA, Abdul-Ghani M: Obesity index that better predict metabolic syndrome: body mass index, waist circumference, waist hip ratio, or waist height ratio. J Obes 2013, 2013:269038

35. Ashwell M, Gunn P, Gibson S: Waist-to-height ratio is a better screening tool than waist circumference and BMI for adult cardiometabolic risk factors: systematic review and meta-analysis. Obes Rev 2012, 13(3):275-286.

36. Elizondo-Montemayor L, Serrano-Gonzalez M, Ugalde-Casas PA, BustamanteCareaga H, Cuello-Garcia C: Waist-to-height: cutoff matters in predicting metabolic syndrome in Mexican children. Metab Syndr Relat Disord 2011, 9(3):183-190.

doi:10.1186/1758-5996-6-32

Cite this article as: Ferreira-Hermosillo et al:: Utility of the waist-to-height ratio, waist circumference and body mass index in the screening of metabolic syndrome in adult patients with type 1 diabetes mellitus. Diabetology \& Metabolic Syndrome 2014 6:32.

\section{Submit your next manuscript to BioMed Central and take full advantage of:}

- Convenient online submission

- Thorough peer review

- No space constraints or color figure charges

- Immediate publication on acceptance

- Inclusion in PubMed, CAS, Scopus and Google Scholar

- Research which is freely available for redistribution 